\title{
Regional Structural Characterization of the Brain of Schizophrenia Patients
}

\author{
Abraham Dubb, Paul Yushkevich, Zhiyong Xie, Ruben Gur, Raquel Gur, and \\ James Gee \\ Departments of Psychiatry and Radiology \\ University of Pennsylvania \\ Philadelphia, PA, USA 19104 \\ \{adubb, pauly2, zxie\}@grasp.cis . upenn.edu \\ $\{$ gur, raquel $\} @ b b l . m e d . u p e n n . e d u$ \\ gee@rad.upenn.edu
}

\begin{abstract}
Abnormal neuro-development and brain structure may play a role in the pathophysiology of schizophrenia. To study morphology and age-related changes in this disease, we started with a set of cranial MRI's of 46 schizophrenia patients and age/gender matched healthy controls. First, we deformed a template brain image to our set of subject images. The Jacobian fields of these deformations were then reduced to sets of 52 normalized region volumes for each subject using a neuro-anatomical atlas. The normalized regional volumes of the control and patient groups were compared using Student's $t$-test. In addition, the age correlation of each region volume was calculated for the two groups. All results were corrected for multiple comparisons using permutation testing. Finally, we used a classifier based on support vector machines and a feature selection method in order to determine our ability to discriminate brains of controls from those of patients. RESULTS: Analysis of the region-integrated Jacobians showed an enlargement of the third ventricle in patients. The age-correlation study demonstrated significant positive correlation in the third ventricle and right thalamus of controls, but not patients. Using an average of 6.5 features, our classifier was able to correctly identify $72 \%$ of patients and $70 \%$ of controls. CONCLUSIONS: In addition to enlargement of the third ventricle, the brains of schizophrenia patients demonstrate a different pattern of age-related changes.
\end{abstract}

\section{Introduction}

The study of schizophrenia has benefited from the advent of high resolution MRI and advanced morphometry techniques. Investigators have reported volumetric differences in multiple regions including the frontal and temporal lobes, ventricles, hippocampus and extra-pyramidal structures [1, 2, 3, 4, 5]. The existence of morphologic differences has motivated evaluation of the developmental course of neuroanatomic measures to assess whether abnormal neuro-development or neuro-degeneration play a role in schizophrenia. Several studies reported regional age-related changes of the brain [6, 7, 8]. While these studies have produced conflicting results, there is a growing consensus that brain plasticity is altered in schizophrenia, underscoring the importance of studying age-related changes in neuroanatomy [6]. 
A rigorous approach to brain volumetry must account for a number of factors. First, there is the issue of normalization: the sizes of internal brain structures are related to overall brain size, however this relationship may be quite variable. Second, discrepancies in the demographic factors of the study groups may have unforseen effects on the results. Finally, volumetric studies must account for multiple comparisons in calculating the significance of observed volumetric differences.

We attempt to address these concerns by using conservative statistical methods to analyze region-based volumes in a population of schizophrenia patients and a set of age/gender matched controls. We generate our region volumes by applying a highresolution atlas to a set of spatially-registered brain MRI's. Although this approach precludes voxel-wise comparisons, region-based analysis reduces the dimensionality of our results, increases the robustness of our findings, and avoids thresholding issues in SPM-style cluster analysis. Finally, we implement a classification algorithm that uses feature selection in order to find that subset of structures which are most discriminating between patient and control groups.

\section{Materials and Methods}

\subsection{Subjects and Data Acquisition}

The Schizophrenia Research Center (SRC) at the University of Pennsylvania maintains a database containing hundreds of prospectively accrued cranial MRI's of psychiatric patients and healthy volunteers. The sample selection procedures have been detailed in Shtasel et al. [9], the MRI acquisition protocol has been described in detail in Gur et al. [10], and results of volumetric segmentation analysis for this sample have been reported [10]. SPGR scans were acquired on a 1.5 T scanner (Signa; General Electric Co., Milwaukee, WI) using the following parameters: flip angle of $35^{\circ}$, repetition time of $35 \mathrm{~ms}$, echo time of $6 \mathrm{~ms}$, field of view of $24 \mathrm{~cm}, 1$ repetition, $1 \mathrm{~mm}$ slice thickness and no interslice gaps. Transaxial images were in planes parallel to the orbitomeatal line, with resolution of $0.9375 \times 0.9375 \mathrm{~mm}^{2}$ [10].

To generate our dataset, we started by selecting the earliest cranial MRI scan of each schizophrenia patient. In order to eliminate the confounding effects of demographics, we chose a set of controls using bipartite graph matching, where each patient was matched to a single normal control of the same gender and of closest possible age. As a result of this matching, mean age, median age, age variance, as well as minimal and maximal ages were very similar in the two groups.

\subsection{Brain Extraction}

The skull, scalp, and other extra-cranial tissues were removed from each image using the Brain Surface Extraction Program, developed by Shattuck et al. [11] In most cases, the software performed perfectly and extracted the brain without error. In several cases, however, additional manual editing was required to remove retained extra-cranial tissue. 
Table 1. Complete listing of all structures included in neuro-anatomical atlas.

\begin{tabular}{|llll|}
\hline corpus callosum & left middle temporal gyrus & right amygdala & right occipital lobe \\
left amygdala & left occipital lobe & right caudate & right occipitotemporal gyrus \\
left caudate & left occipitotemporal gyrus & right cerebellar hemisphere & right parahippocampal gyrus \\
left cerebellar hemisphere & left parahippocampal gyrus & right csf & right postcentral gyrus \\
left csf & left postcentral gyrus & right globus pallidus & right precentral gyrus \\
left globus pallidus & left precentral gyrus & right grey matter & right putamen \\
left grey matter & left putamen & right hippocampus & right superior frontal gyrus \\
left hippocampus & left superior frontaman pdl gyrus & right inferior frontal gyrus & right superior parietal lobule \\
left inferior frontal gyrus & left superior parietal lobule & right inferior parietal lobule & right superior temporal gyrus \\
left inferior parietal lobule & left superior temporal gyrus & right inferior temporal gyrus & right supramarginal gyrus \\
left inferior temporal gyrus & left supramarginal gyrus & right lateral ventricle & right thalamus \\
left lateral ventricle & left thalamus & right middle frontal gyrus & right white matter \\
left middle frontal gyrus & left white matter & right middle temporal gyrus & third ventricle \\
\hline
\end{tabular}

\subsection{Template Image and Atlas Creation}

We obtained a simulated T1 $1 \mathrm{~mm}$ isotropic brain MRI and accompanying tissue masks from the Brainweb website 1 [12 13] . This brain image served as our template image and was the basis for our atlas creation. We used a variety of methods to map out a total of 52 different regions on the brain. For the subcortical structures, we used a combination of manual slice-by-slice painting and automatic 3D level-set based segmentation facilities provided by the open source ITK-SNAP software 2 . In order to map out the cortical areas on the brain surface, we employed a three step method. First, a series of curves that separate the various regions of interest were drawn by an expert on the surface of the grey matter. Second, these curves were projected onto the surface of the white matter using a combination of Euclidean distance transform and Dijkstra's shortest path algorithm, resulting in series of ribbons that penetrate the grey matter hull. Finally, the ribbons were rasterized and automatic segmentation was used to fill in each region bounded by the ribbons. Figure 1 shows several projections of our atlas. Table 1 lists all structures included in the atlas.

\subsection{Image Registration}

The registration method we applied is a spline-based extension to Thirion's Demons technique [14]. It uses optical flow to determine the correspondence of voxels which exhibit sufficiently large intensity gradients. Based on the estimated, sparse correspondences, a B-spline function of the correspondences over the whole brain volume is determined using weighted scattered data approximation. This two-step algorithm is applied over multiple resolution levels in conventional coarse to fine fashion: both the resolution of the images and the number of spline control parameters are simultaneously adjusted. Specifically, starting with B-spline functions that have a small number of parameters, the algorithm is iterated to match the coarse features of the images. The result is used to initialize the registration at the next resolution level, where the number of spline parameters is increased to allow alignment of the finer features that are apparent in the higher resolution images. This strategy provides a way to incrementally refine the registration and improves the robustness of the method.

\footnotetext{
${ }^{1}$ Available from http://www.bic.mni.mcgill.ca/brainweb/

${ }^{2}$ Available from http://www. cognitica.com/snap
} 

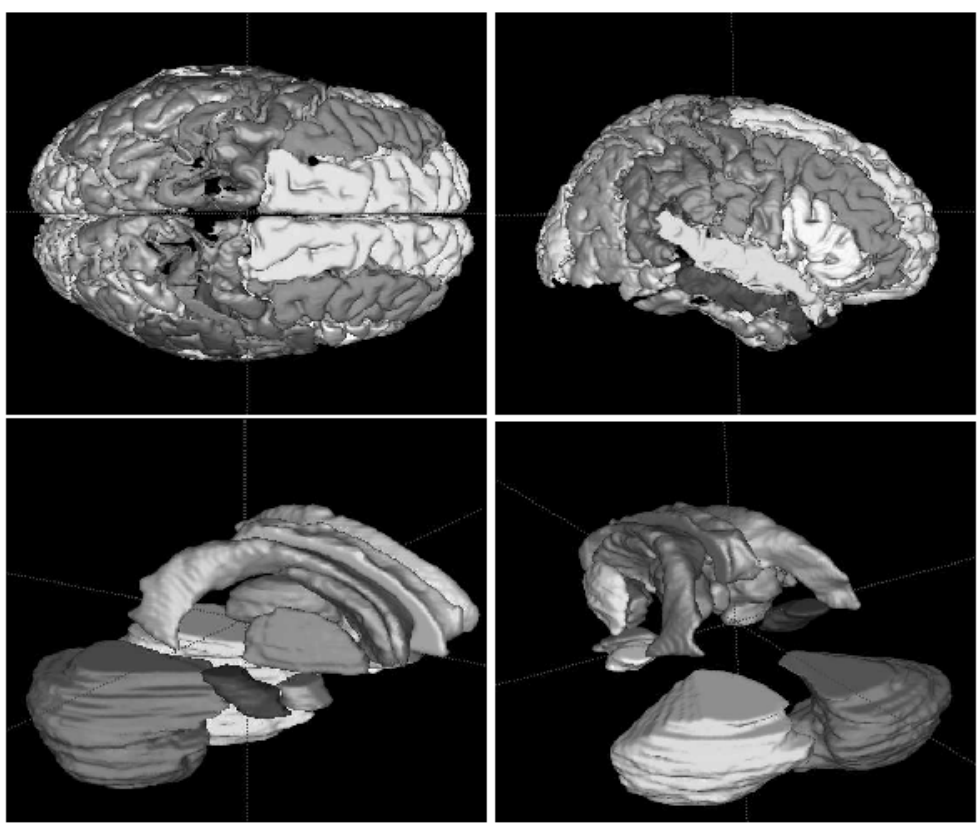

Fig. 1. Internal and external projections of the atlas used for our region-of-interest calculations.

This B-spline registration method has been validated on a separate labeled image set (unpublished data), and is capable of yielding overlap ratios of greater than $95 \%$ for lobar structures (frontal lobe, temporal lobe, etc) and close to $80 \%$ for small structures such as the amygdala and hippocampus. We used this registration method for the generation of our deformation fields and corresponding Jacobian images as described below.

\subsection{Statistical Analysis of the Jacobian}

We registered our template brain to each subject brain, generating a deformation field for each subject. The Jacobian of the deformation field was computed at each voxel, giving us a voxel-wise size comparison between subject and template. In each subject, the Jacobian was integrated over each region and normalized by total brain Jacobian, yielding a set of 52 normalized structure volumes. These structure volumes served as features in subsequent statistical studies.

Statistical analysis involved applying both hypothesis testing and classification in order to determine whether schizophrenia is associated with volumetric changes in the selected regions. In the hypothesis testing experiments we tested the equality of means for each feature using Student's $t$-test, as well as the within-group correlation with age, denoted $R$, using Fisher's one-sample $z$-test and inter-group difference in age correlation using the two-sample $z$-test. In order to account for the effect of multiple testing on the 
significance level of the overall findings, the significance level of each individual test was corrected using random permutations. In applying permutation testing we followed the techniques of Nichols and Holmes [15], repeatedly reassigning group labels to subjects at random, applying a given hypothesis test to each of the features, and recording the maximum value of the corresponding statistic ( $t$ or $z$ ). In the resulting histogram of maximal statistics, we found the fraction of random experiments, denoted $p^{*}$, for which the maximal value of the statistic exceeded the observed statistic for a given comparison.

To reinforce the results of hypothesis testing, we applied classification to gauge how well the region-based features can be used to correctly determine the group membership of subjects. We use a classifier based on support vector machines (SVM) and the feature selection method of Bradley and Mangasarian [16]. This classifier computes a linear separation boundary between the classes in a low-dimensional subspace of the feature space. This method has an added advantage of identifying the structures that are most relevant for classification. The method has one parameter that influences the dimensionality of the classification subspace, i.e. the number of stuctures used. We calculated the generalization ability of the classifier for 20 different parameter settings using the leave-one-out method.

\section{Results}

A total of 92 subjects were analyzed, 46 patients and 46 matched controls, with average ages of $30.8 \pm 10.4$ years and $31.0 \pm 10.4$ years, respectively. Both groups were composed of 30 males and 16 females. The ten most significant region volume comparisons are displayed in table 2] After correction for multiple comparisons, the third ventricle was the only structure which retained significance $\left(p^{*}=0.065\right)$, and it was larger in patients. Table 3 shows the five most significant correlations for controls and patients. The third ventricle and right thalamus were significantly positively correlated with age in controls $\left(p^{*}=0.0124,0.0191\right.$, respectively). In patients, however, the most age-correlated region was the right cerebrospinal fluid volume (CSF) and had a $p$-value of only 0.1263 . The CSF region is defined as the combination of the brain ventricles and the external fluid filling the cortical sulci. There were no significant findings for the two-sample Fisher's $z$ comparison of age-correlations between the two groups.

Our SVM-based classifier achieved the lowest cross-validation error rate using a feature selection parameter that yielded an average of 6.5 features. Using this classifier, we correctly identified $72 \%$ of patients, and $70 \%$ of controls. The most frequently used structures in this classifier were the third ventricle (93\% of experiments), left middle temporal gyrus (85\%), and left superior parietal lobule $(73 \%)$.

\section{Discussion}

We applied a neuro-anatomical atlas to a set of spatially registered images to study volumes and age-correlation of brain structures in schizophrenia. We validated our hierarchical non-rigid registration method on a separate expert-labeled dataset and found it to be superior to several other warping algorithms. Our study benefits from the quality of our atlas, which we created specifically for our template image. The cortical regions 
Table 2. Partial listing of mean normalized region volume comparisons of patients vs. controls. $p$ refers to uncorrected $p$-value for Student's $t$-test comparison. $p^{*}$ denotes the $p$-value corrected for multiple comparisons through permutation testing.

\begin{tabular}{|l|c|c|c|c|}
\hline Region & Controls & Patients & $p$ & $p^{*}$ \\
\hline third ventricle & 0.00067 & 0.00072 & 0.001 & 0.065 \\
right middle frontal gyrus & 0.01475 & 0.01416 & 0.014 & 0.454 \\
left superior parietal lobule & 0.00829 & 0.00794 & 0.016 & 0.485 \\
right lateral ventricle & 0.00555 & 0.00609 & 0.054 & 0.892 \\
right thalamus & 0.00319 & 0.00330 & 0.078 & 0.961 \\
right superior parietal lobule & 0.00886 & 0.00852 & 0.078 & 0.961 \\
left caudate & 0.00190 & 0.00198 & 0.083 & 0.968 \\
right precentral gyrus & 0.00544 & 0.00528 & 0.085 & 0.971 \\
left postcentral gyrus & 0.00687 & 0.00667 & 0.086 & 0.972 \\
left lateral ventricle & 0.00588 & 0.00639 & 0.088 & 0.974 \\
\hline
\end{tabular}

Table 3. Partial listing of region volume-age correlation in controls (left) and patients (right). $R$ refers to the Pearson correlation coefficient. $p$ and $p^{*}$ are the uncorrected and corrected scores for the Fisher's $z$ statistic.

Controls:

\begin{tabular}{|l|c|c|c|}
\hline Region & $R$ & $p$ & $p^{*}$ \\
\hline third ventricle & 0.53 & 0.0001 & 0.0124 \\
right thalamus & 0.51 & 0.0002 & 0.0191 \\
left thalamus & 0.41 & 0.0046 & 0.2010 \\
right white matter & -0.39 & 0.0074 & 0.2906 \\
right hippocampus & 0.33 & 0.0253 & 0.6525 \\
\hline
\end{tabular}

Patients:

\begin{tabular}{|l|c|c|c|}
\hline Region & $R$ & $p$ & $p^{*}$ \\
\hline right CSF & 0.43 & 0.0025 & 0.1263 \\
right inf. frontal gyrus & -0.42 & 0.0032 & 0.1577 \\
third ventricle & 0.42 & 0.0036 & 0.1732 \\
right thalamus & 0.33 & 0.0268 & 0.6836 \\
right occipital lobe & -0.30 & 0.0438 & 0.8415 \\
\hline
\end{tabular}

were generated from surface-drawn boundaries, rather than slice-by-slice painting. In addition, the use of 3D level-set segmentation for certain structures (e.g. ventricles) ensures high resolution and lack of inter-slice shift.

By using an atlas to define our features, we accomplish data-reduction in an anatomically meaningful way. Reducing each subject to a set of 52 region volumes allows us to apply permutation-based statistical techniques and classification algorithms that would otherwise be impractical. We employed rigorous and conservative statistical methods to avoid false positives and the confounding effects of subject demographics.

Our results showed a significance difference in only one region volume, the third ventricle. The lateral ventricles, which are generally believed to be larger in schizophrenia [17], are in the "top ten" of our results, but fail to achieve significance. Our methods can miss structural differences if they fall between regions defined by our atlas, or if such differences are averaged out by relatively large region sizes. Future work, therefore, includes development of a more detailed and hierarchical atlas. Correlations between age and volume in the two groups show no significant differences in the two-sample Fisher's $z$ comparison, but suggest a different pattern of brain maturation; the third ventricle is highly age-correlated in controls, yet is smaller than in schizophrenia. The lack of agecorrelation in schizophrenia of this structure suggests that this structure's age-related 
expansion occurred earlier in life and has plateaued, suggesting a neuro-developmental process.

The ability of our classification algorithm to achieve over $70 \%$ accuracy certainly suggests that morphologic differences exist between the subgroups, but that such differences are far from being robust enough to permit perfect discrimination. Interestingly, the left middle temporal gyrus was the second most frequently used feature, yet was ranked 12th in the direct volume comparison. This finding emphasizes the nature of schizophrenia as being a constellation of fairly slight morphologic changes.

The subtlety of our reported findings emphasize the need for high quality image analysis tools as well as a sufficient sample size. Further characterization of the neurodegenerative versus neuro-developmental aspects of schizophrenia will aid in future diagnosis and treatment.

Acknowledgements. This work was supported in part by the USPHS under grants P30-NNC, LM-03504, MH-62100, MH60722, MH-19112, MO1RR0040, AG-15116, AG-17586, and DA-14418.

\section{References}

1. RE Gur, PE Cowell, A Latshaw, BI Turetsky, RI Grossman, SE Arnold, WB Bilker, and RC Gur. Reduced dorsal and orbital prefrontal gray matter volumes in schizophrenia. Arch Gen Psychiatry, 57(8):761-8, 2000.

2. RE Gur, BITuretsky, PE Cowell, C Finkelman, V Maany, RI Grossman, SE Arnold, WB Bilker, and RC Gur. Temporolimbic volume reductions in schizophrenia. Arch Gen Psychiatry, 57(8):769-75, 2000.

3. W Cahn, HE Pol, M Bongers, HG Schnack, RC Mandl, NE Van Haren, S Durston, H Koning, JA Van Der Linden, and RS Kahn. Brain morphology in antipsychotic-naive schizophrenia: A study of multiple brain structures. Br J Psychiatry - Suppl, 43:s66-72, 2002.

4. RE Gur, V Maany, D Mozley, C Swanson, W Bilker, and RC Gur. Subcortical MRI volumes in neuroleptic-naive and treated patients with schizophrenia. Am J Psychiatry, 155:1711-1717, 1998.

5. MS Keshavan, D Rosenberg, JA Sweeney, and JW Pettegrew. Decreased caudate volume in neuroleptic-naive psychotic patients. Am J Psychiatry, 155(6):774-8, 1998.

6. LE Delisi. Regional brain volume change over the life-time course of schizophrenia. $J$ Psychiatr Res, 33:535-541, 1999.

7. BT Woods, D Yurgelun-Todd, FM Benes, FR Frankenburg, Jr Pope HG, and J McSparren. Progressive ventricular enlargement in schizophrenia: comparison to bipolar affective disorder and correlation with clinical course. Biol Psychiatry, 27(3):341-52, 1990.

8. JL Rapoport, J Giedd, S Kumra, L Jacobsen, A Smith, P Lee, J Nelson, and S Hamburger. Childhood-onset schizophrenia progressive ventricular change during adolescence. Arch Gen Psychiatry, 54(10):897-903, 1997.

9. DL Shtasel, RE Gur, PD Mozley, J Richards, MM Taleff, C Heimberg, F Gallacher, and RC Gur. Volunteers for biomedical research: recruitment and screening of normal controls. Arch Gen Psychiatry, 48:1022-1025, 1991.

10. RC Gur, B I Turetsky, M Matsui, M Yan, W Bilker, P Hughett, and RE Gur. Sex differences in brain gray and white matter in healthy young adults. J Neurosci, 19:4065-4072, 1999. 
11. DW Shattuck, SR Sandor-Leahy, KA Schaper, DA Rottenberg, and RM Leahy. Magnetic resonance image tissue classification using a partial volume model. Neuroimage , 13(5):85676, 2001.

12. RK-S Kwan, AC Evans, and GB Pike. An extensible MRI simulator for post-processing evaluation. In Visualization in Biomedical Computing, volume 1131, pages 135-140. SpringerVerlag, Berlin, 1996.

13. DL Collins, AP Zijdenbos, V Kollokian, JG Sled, NJ Kabani, CJ Holmes, and AC Evans. Design and construction of a realistic digital brain phantom. IEEE Transactions on Medical Imaging, 17:463-468, 1998.

14. JP Thirion. Image matching as a diffusion process: an analogy with Maxwell's demons. Med Image Anal, 2(3):243-60, 1998.

15. TE Nichols and AP Holmes. Nonparametric analysis of pet functional neuroimaging experiments: A primer. Human Brain Mapping, , 15:1-25, 2001.

16. PS Bradley, OL Mangasarian, and WN Street. Feature selection via mathematical programming. INFORMS Journal on Computing, 10:209-217, 1998.

17. R McCarley, C Wible, M Frumin, Y Hirayasu, J Levitt, I Fischer, and M Shenton. Mri anatomy of schizophrenia. Biol. Psychiatry, 45:1099-1119, 1999. 